\title{
Gpi17p does not stably interact with other subunits of Glycosylphosphatidylinositol transamidase in Saccharomyces cerevisiae
}

Yonghua Zhu ${ }^{l}$, Patrick Fraering ${ }^{1,2}$, Christine Vionnet and Andreas Conzelmann

Department of Medicine/Biochemistry, University of Fribourg, Switzerland

Corresponding author e-mail: andreas.conzelmann@unifr.ch

Dr. A. Conzelmann, Division of Biochemistry, Chemin du Musée 5, CH-1700 Fribourg,

Switzerland. Tel. ++4126300 8630. Fax ++41263009735.

\footnotetext{
${ }^{1}$ The first two authors contributed equally to this work

${ }^{2}$ Present address : Center for Neurologic Diseases, Brigham and Women's Hospital and Harvard Medical School, Boston, MA 02115
}

RUNNING TITLE: Yeast glycosylphosphatidylinositol transamidase

Keywords: GPII7 ; Glycosylphosphatidylinositol ; transamidase ; saccharomyces ; protein stability ; unfolded protein response 


\section{Summary}

Homologues of Gpi8p, Gaa1p, Gpi16p, Gpi17p and Cdc91p are essential components of the GPI transamidase complex that adds glycosylphosphatidylinositols (GPIs ${ }^{1}$ ) to newly synthesized proteins in the ER. In mammalian cells these five subunits remain stably associated with each other in detergent. In yeast we find no stable, stoichiometric association of Gpi17p with the Gpi8p-Gpi16p-Gaa1p core in detergent extracts. Random and site directed mutagenesis generated mutations in several highly conserved amino acids but did not yield non-functional alleles of Gpi17p and a saturating screen did not yield any dominant negative alleles of Gpi17p. Moreover, Gpi8p becomes unstable when any one of the other subunits is depleted, whereas Gpi17p is slightly affected only by the depletion of Gaalp. These data suggest that yeast Gpi17p may be able to exert its GPI anchoring function without interacting in a stable and continuous manner with the other GPI-transamidase subunits. Shutting down ER associated and vacuolar protein degradation pathways has no effect on the levels of Gpi17p or other transamidase subunits.

\section{Abbreviations}

\footnotetext{
${ }^{1}$ Abbreviations used: BCS bathocuproinedisulfonic acid; GPI, glycosylphosphatidylinositol; NP-40, Nonidet P-40; TMD, transmembrane domain; UPR, unfolded protein response; wt, wild type.
} 


\section{Introduction}

Precursors of GPI anchored proteins have a classical signal sequence for import into the ER at their N-terminus and a GPI anchoring signal at their C-terminus; the C-terminal signal is necessary and sufficient to direct GPI addition [1]. The C-terminal GPI anchoring signal is recognized and removed by a GPI transamidase, which replaces it by a preformed GPI. GPI anchoring signals are composed of a C-terminal hydrophobic domain, which is separated by a short hydrophilic spacer from the cleavage/attachment site ( $\omega$ site) [2-4]. Genetic and biochemical approaches have so far identified 5 proteins that encode transamidase subunits [5-9]. All 5 proteins are essential in yeast and all are required for the nucleophilic attack on the $\omega$ site residue $[8,9]$. GAAl encodes a $70 \mathrm{kDa}$ ER protein with an uncleaved N-terminal signal sequence, a large, hydrophilic, lumenal domain, followed by several transmembrane domains (TMD) and an ER retrieval signal on its extreme C-terminus, that however is not functional as it is oriented towards the ER lumen $[8,10]$. Its last TMD is required to bind the GPI lipid substrate into the complex [11]. GPI8 and GPI16 encode type I ER membrane proteins with large lumenal domains and a single TMD [6-8]. Gpi8p has 25 - 28\% homology to a family of cysteine proteinases and the mutation of Cys and His predicted to be active site residues by sequence comparison with related proteases yields non-functional GPI8 alleles [12-14]. Moreover, purified trypanosomal Gpi8p was shown to contain proteolytic activity towards appropriate peptide substrates [15]. Gpi16p is linked through a functionally important disulfide bridge to Gpi8p [16] and has been proposed to form a funnel that gates the access of proteins to the active site of the Gpi8p protease and thus contributes to the specificity of the GPI anchor addition [17]. Gpi17p is predicted to be mainly lumenal with its N- and Cterminal ends both being cytosolic. Cdc91p is a very hydrophobic protein with many TMDs [9, 18]. While Gaa1p, Gpi8p and Gpi16p are common to all eukaryotic organisms, Gpi17p and Cdc91p are absent from trypanosomatids, which latter possess TTA1 and TTA2 in their stead [19]. 


\section{Materials and Methods}

\section{Strains, Growth Conditions and Materials}

Saccharomyces cerevisiae strains used are listed in Table 1. TAP tagged strains [20] were obtained from OPEN BIOSYSTEMS, Huntsville, AL.

Cells were grown on rich medium (YPD) or minimal media (SD or SG) [21] containing 2\% glucose (D) or galactose (G) as a carbon source and uracil (U), adenine (A) and amino acids (aa) as required at $30^{\circ} \mathrm{C}$ but lacking inositol. Peptides were synthesized by ALTA Bioscience, University of Birmingham, UK. Oligonucleotide synthesis and DNA sequencing services were provided by MICROSYNTH, Balgach, Switzerland.

\section{Construction of yeast vectors}

The open reading frame of GPII7 and its transcription terminator region was placed behind the GAL1 promoter and inserted into YCplac111 using the same restriction sites and procedures as used to construct YCplac111-GAL1-GPI16 [7], thereby generating YCplac111GAL1-GPI17. GST was amplified using primers 5'-AACCCCGGAT-

CCATGTCCCCTATACTAGGTTATTGG-3' and 5'- GGACATGTCGACACGCGGAACCAGATCC-3' on the template pFA6a-His3MX6-PGAL1-GST [22]. This PCR fragment was inserted at the N-terminus of GPI17 between the BamHI and Sal1 site of YCplac111-GAL1GPI17.

For construction of plasmid pCUP1-GPI17, two overlapping parts of the GPI17 open reading frame were amplified by PCR using 5'-CTGTGAATTCTGATCAAAAAAAATGTCCAATGCAAATCTAAG-3' and 5'-GTTATTCTGCAGTGACTCAATG-3' for one PCR reaction, 5'- CATTGAGTCACTGCAGAATAAC-3' and 5'-ACAAAGTCGACAACTCATAATTCATCTTCACCATC- 3' for the second reaction. The two PCR fragments were digested with EcoRI/PstI and PstI/ SalI, respectively, and were inserted into the multiple cloning site of the CUP195 vector [23]. New gpil7 alleles were produced by random PCR mutagenesis of three conserved fragments of Gpi17p, which could be removed from CUP195-GPI17 by cutting at unique restriction sites. Mutagenized fragments containing the sequences going from residue 7 to 40,206 to 247 and 421 to 528 were inserted in the appropriately opened CUP195-GPI17 vector and were transfected into wild type (wt) yeast cells (W303-1B). Transformants growing on $100 \mu \mathrm{M}$ BCS were replica plated onto plates containing $200 \mu \mathrm{M} \mathrm{Cu}^{2+}$ and clones 
not growing on the latter were chosen for further study. A K28A mutation was introduced into Gpi17p by crossed PCR with one fragment generated with primers 5'-

AACCGGTACCATATAATGTCCAATGCAAATCTAAG-3' and 5'-

AATTTATTGGTAGTGATGCTCTATAGACTGTAGTCAGCGCGTACCACAGTGGAAC ACCTA -3', another generated with 5'-TATAGAGCATCACTACCAATAAATT-3' and 5'GACCAAGGGACCCGTTGC-3'. The PCR product of a crossed PCR reaction using these two fragments as templates was digested with KpnI/EcoRI and inserted into pYES2 (INVITROGEN) thus generating pYES2-GPI17. An analogous strategy was used to generate the R34A mutation. The thus generated R34A and K28A gpil7 alleles in pYES2 were transfected into the haploid wt (W303-1B) and the diploid Y24270 gpi17::kanMX4/GPI17 strains. All constructs were verified by DNA sequencing.

\section{Cell extraction and Western blotting}

Antibodies against Gpi8p and Gpi16p have been described [6, 7]. Antibodies against Gaa1p and Gpi17p were obtained by immunizing rabbits with octameric peptides corresponding to residues 311-339 of Gaa1p and 43-70 of Gpi17p. Antibodies were used after affinity purification on the same peptide. Two His6-tagged fragments of hydrophilic parts of Gpi17p corresponding to amino acids 64 to 260 and 343 to 470 of yeast GPII7 were introduced into the bacterial expression vector pET15b. Gpi17p fragments were extracted from bacteria, affinity purified, injected into rabbits and coupled to Sepharose for affinity purification of the antibodies. Proteins were extracted by incubating cells for $5 \mathrm{~min}$ in $\mathrm{NaOH}$ and boiling at $95^{\circ} \mathrm{C}$ in reducing sample buffer [24].

\section{Affinity purification of transamidase complexes}

Membranes were obtained and extracted with $1 \%$ digitonin as described for affinity purification of the transamidase complex containing GST-Gpi8p [7]. Affinity chromatography of complexes containing GST-tagged Gpi17p was done exactly as described for the complex containing GST-Gpi8p [7]. Pull down experiments of complexes containing a TAP-tagged transamidase subunit were done in an analogous manner using human IgG Sepharose beads [20]. Beads were washed three times in the IPP150 buffer (10mM Tris-HCl, pH8.0, 150mM $\mathrm{NaCl}, 0.2 \%$ digitonin) by sedimenting them at $20 \mathrm{~g}$ for $5 \mathrm{~min}$ and then treated with reducing SDS-PAGE sample buffer, $5 \mathrm{~min}$ at $95^{\circ} \mathrm{C}$. The samples were processed for Western blotting and probed with anti-protein A antibody, or other antibodies that could bind through their $\mathrm{Fc}$ 
moiety to the TAP tag. In some experiments $1 \%$ of Nonidet P-40 (NP-40) was used instead of digitonin.

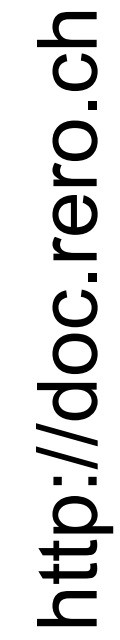




\section{Results}

\section{The bulk of Gpi17p is not associated with the yeast transamidase core complex.}

The affinity purified mammalian transamidase complex has been reported to contain PIG$\mathrm{S} / \mathrm{Gpi} 17 \mathrm{p}$ appearing as a band of similar intensity as the other four subunits on silver stained gels $[8,16]$. The use of a fully functional GST-Gpi8p construct allowed to isolate a similar protein complex in yeast, which however only contained three subunits, namely GST-Gpi8p, Gpi16p and Gaa1p. No other subunits were detected on the silver stained SDS/PAGE gel [7]. To verify if this complex nevertheless contains Gpi17p, we generated rabbit anti-Gpi17p antibodies. They detect bands at 63 and $50 \mathrm{kDa}$, which gain in intensity, when Gpi17p is overexpressed (Fig. 1A). After treatment with Endoglycosidase H, the $63 \mathrm{kDa}$ band runs at about $51 \mathrm{kDa}$. This is less than the expected molecular mass of $60.8 \mathrm{kDa}$ (Fig. 1B), but the high mobility may be due to the low pI (4.5) of Gpi17p. According to http://www.cbs.dtu.dk/services/SignalP/, the N-terminal hydrophobic leader peptide of yeast Gpi17p is not cleaved, and, as shown experimentally below, the $\mathrm{N}$ - as well as the Cterminally tagged Gpi17p protein can be detected using tag specific antibodies (Figs. 3B and 3C). The $63 \mathrm{kDa}$ glycoprotein behaved as an integral membrane protein as expected from its primary sequence (Fig. 1C).

Anti-Gpi17p antibodies allowed to follow Gpi17p during the purification of the GST-Gpi8p yeast transamidase complex. We detected Gpi17p in the material that had run through the glutathione-Sepharose affinity column, but not in the material that had been retained and eluted (Fig. 2A). Next we used anti-Gpi17p antibodies to probe by Western blot the transamidase complex separated on blue native gel electrophoresis (Fig. 2B). There, all Gpi17p was found in a high molecular weight complex, close to the $430-650 \mathrm{kDa}$ region, where previous experiments had detected Gpi8p [7]. However, close inspection showed that the bulk of Gpi17p was contained in a complex with slightly lower molecular mass than the Gpi8p-containing complex (Fig. 2B, lanes 3-6 and 3' -6'). These results suggested that the bulk of Gpi17p is not present in the same complex as Gpi8p, or, to the least, that the interaction of Gpi17p with Gpi8p-Gpi16p-Gaa1p is to weak to survive solubilization in digitonin and subsequent electrophoresis. The fact that the purified GST-Gpi8p-complex had 
almost the same molecular weight in blue native electrophoresis as the same complex in the crude extract [7] also makes it unlikely that a Gpi17p subunit would be associated with the Gpi8p-Gpi16p-Gaa1p complex and would subsequently be lost during purification.

One possible reason for the absence of Gpi17p in the purified GST-Gpi8p complex (Fig. 2A, lane 5) may have been steric hindrance, as we used a N-terminal GST tag on Gpi8p, while the mammalian complex was purified using a Gpi8p modified by a C-terminally attached FLAGGST tag [8]. Thus, to further test if Gpi17p is present in the same complex as Gpi8p, we solubilized wt cell membranes in $1 \%$ digitonin and fractionated the lysate by ultracentrifugation over a glycerol density gradient. We found that most of Gpi8p and Gpi16p was present in fractions of intermediate density containing little Gpi17p (Fig. 3A). The bulk of Gpi17p stayed in fractions of low density (fractions $1-5$ ) and only a minor amount of Gpi8p could be found together with Gpi17p in fractions 4 and 5 (Fig. 3A). Thus, most of Gpi17p was not found associated with the Gpi8p-Gpi16p complex in a situation, where none of the proteins carried any tag and all subunits were present at their physiological levels since encoded by the endogenous, chromosomal genes. A recent genome wide analysis of protein expression levels in yeast showed that a cell growing on rich media contains 1560, 1680 and 7520 copies of Gpi8p, Gpi16p and Gpi17p, respectively [20]. The relative excess of Gpi17p over other subunits may explain why some $80 \%$ of Gpi17p was not found in the same fractions as Gpi8p and Gpi16p (Fig. 2A). To explore this further we adopted a more rapid purification procedure and used strains containing TAP-tagged Gpi17p [20] or GST-tagged Gpi17p. In these strains we checked if we could co-precipitate Gpi8p and Gpi16 with tagged Gpi17p (Figs. 3B to 3D). If each transamidase contained a Gpi17p subunit, one expected that quantitative precipitation of Gpi17p would quantitatively precipitate Gpi8p and Gpi16p and that the ratio of Gpi8p over Gpi17p, and Gpi16p over Gpi17p were the same in the lysate and the precipitate. The Western blots however clearly showed that the ratios of Gpi8p over Gpi17p, and Gpi16p over Gpi17p were much lower after an affinity purification step than in the starting digitonin extracts (Fig. 3B, 3C). In NP-40 extracts, Gpi17p-TAP did not coprecipitate any Gpi16p (Fig. 3D) or Gpi8p (not shown). Taken together, these data suggest that some Gpi17p may be loosely associated with the yeast transamidase complex but that a majority of complexes does not contain Gpi17p. This is in marked contrast to the mammalian complex, which contained stoichiometric amounts of PIG-S/Gpi17p after having been purified from a NP-40 lysate by a two-step affinity purification $[8,16]$. 
2. Mutation of conserved residues of GPII7 does not lead to a dominant negative growth phenotype.

To demonstrate that an interaction of Gpi17p with other subunits is required for GPI anchoring we tried to generate dominant negative mutant alleles of Gpi17p, as the existence of such alleles is an indicator of essential protein-protein interactions and also can pinpoint catalytically important residues. In preliminary experiments we found that the induction of wt GPI17, expressed from a multicopy vector under the control of the inducible CUP1 promoter, leads to a dramatic overexpression of Gpi17p without blocking cell growth. We used error prone PCR to make three libraries of GPII7 alleles mutated in either one of the three most conserved regions extending from amino acids 7 to 40, 206 to 247 and 421 to 528, respectively. In order to make the screen saturating and based on the known mutation frequency of the procedure we analyzed 2000, 2000, and 4000 clones from the 3 libraries. Out of these 8000 we obtained 26 clones that grew well on plates containing the copper chelator BCS, but did not grow in the presence of copper and stably maintained this phenotype. However, when the plasmids were retransfected into fresh wt cells, none of them was able to reproduce the dominant negative growth effect on copper. Their sequencing showed that several of them had mutations in conserved residues, many had mutations in non-conserved residues, and others had no mutation in and around the mutated region (700 base pairs were sequenced). To test the functionality of the generated gpil7 alleles, 7 plasmids were introduced into the diploid Y24270 gpil7::kanMX4/GPI17 strain, which then was sporulated. Four viable spores were obtained from diploids harboring G22S, W26R, Y27H/I42T, A35T/C13Y, P38L or F222S, and G418 resistant clones grew normally on $200 \mu \mathrm{M}$ copper as well as $100 \mu \mathrm{M}$ BCS. (It should be noted that the CUP1 promoter cannot be repressed very efficiently on BCS). On the other hand, the quadruple mutant L212F/Y215F/F219S/T230I proved to be nonfunctional. In view of this we decided to deliberately introduce mutations in two other conserved residues of Gpi17p. Only 7 residues are absolutely conserved amongst the 11 full length PIG-S homologues analyzed by Birgit Eisenhaber et al. at http://mendel.imp.univie.ac.at/SEQUENCES/gpi-biosynthesis/. Of those, only R34 is hydrophilic and located outside the TMDs. Mutations in R34 and the almost as well conserved K28 were generated by oligonucleotide directed mutagenesis and introduced behind the GAL1 promoter into a multicopy plasmid. Gpil7 alleles R34A and K28A fully complemented gpil7 $\Delta$ cells, even when they were grown under repressing conditions 
(glucose media) and these alleles had no dominant negative phenotype when overexpressed in wt cells. We suspected that plasmids rescuing gpi17 $\Delta$ strains could have reverted due to meiotic recombination and conversion. Therefore rescuing plasmids harboring GPII7 with either a G22S, W26R, A35T/C13Y, P38L, K28A or R34A mutation were isolated again from the haploid gpil7s strains that had been obtained through sporulation of the plasmid transfected Y24270 gpi17::kanMX4/GPI17 diploid strains. Plasmids were also reisolated from two GPI17 clones harboring L212F/Y215F/F219S/T230I. Sequencing of an isolate from two different gpil7 $\Delta$ strains for each mutation showed that all plasmids had preserved their original mutation and had not been corrected by meiotic recombination during sporulation. It was also verified by PCR that all these gpil7A cells, which had been rescued by a mutant allele of GPI17, did not harbor any GPII7 under its natural promoter due to chromosome duplication. Thus, although conserved, G22, W26, K28, R34, A35, P38 and I42 are not important for the function of Gpi17p (Table 2).

\section{Gpi8p is very sensitive to depletion of other subunits.}

A potential interaction between Gpi17p and other subunits was further investigated by testing the stability of Gpi17p when other subunits are depleted or vice versa. For this, we constructed strains, in which one subunit at a time was expressed from the GAL1 promoter rather than its own promoter allowing cells to be depleted of that subunit on glucose. As can be seen in Fig. 4, it proved difficult to deplete Gpi17p (lane 16), while the other 3 subunits could be depleted much more efficiently (lanes 4, 8, 12). Depletion of Gpi17p somewhat affected the stability of Gpi8p, but not of the other subunits, and Gpi17p itself was destabilized only by the depletion of Gaa1p. Gpi8p was quite sensitive and readily destabilized when one of the other subunits was depleted (Fig. 4, compare the levels of Gpi8p in lanes 8, 12 and 16 with those in lanes 5, 9 and 13, respectively). On the other hand, Gpi16p and Gaalp were not very much influenced by the depletion of other subunits (Fig. 4, compare the levels of Gpi16p in lanes 4, 8 and 16 with those in lanes 1,5 and 13, respectively and the levels of Gaa1p in lanes 4 and 12 with those in lanes 1 and 9, respectively). In interpreting the results it has to be kept in mind that the depletion of a GPI transamidase subunit and the concomitant deficiency of GPI anchor addition induces an unfolded protein response (UPR)[25], and that the overexpression of a transamidase subunit potentially does the same $[26,27]$. Thus, depletion and overexpression of a transamidase subunits may lead to the 
induction of GAAl and GPI16, the mRNAs for which are upregulated during the UPR while those for GPII7 and GPI8 are not [28]. When going from overexpression to depletion of Gpi8p or Gpi16p one indeed observes an up-down-up profile for the classical UPR target Wbp1p (lanes 1 - 4, 9-12) and a similar profile is seen for Gaa1p during depletion of Gpi8p or Gpi16p, and is also seen for Gpi16p during the depletion of Gpi8p (Fig. 4). Interestingly, in several experiments we saw that the overexpression of Gpi17p on galactose in FBY7310 leads to a significant, concomitant increase of Gaa1p (Fig. 4, compare the Gaalp levels in lane 13 with lanes 1,5, and 9); this observation awaits further investigation.

\section{Degradation of transamidase subunits by ERAD and vacuolar hydrolases.}

Gpi8p subunits, when overexpressed in a wt background seem to be degraded more rapidly than the Gpi8p contained within the transamidase complex [7] and here we find that transamidase subunits are downregulated when other subunits are depleted below their physiological levels. This suggests that they can become targets of vacuolar or ER associated protein degradation (ERAD) pathways and these pathways may also degrade supernumerary subunits under physiological conditions. If this were true, those subunits, which are stabilized through complex formation ought to be present in higher amounts if the relevant degradation pathway is not functional. As can be seen in Fig. 5, the abundance of transamidase subunits was assessed by Western blotting in various mutants blocking either Hrd3p- or Doa10pdependent ERAD or the vacuolar degradation pathways [29]. No major increases of subunits can be observed and the minor increases we observed could not be confirmed in repeat experiments. It thus would appear that ERAD and vacuolar degradation do not constitutively eliminate any supernumerary transamidase subunits under physiological conditions, suggesting that transamidase subunits are made in near to stoichiometric amounts. 


\section{Discussion}

A recent genome wide analysis of protein expression levels in yeast showed that a cell growing on rich media contains 1560, 1680 and 7520 copies of Gpi8p, Gpi16p and Gpi17p, respectively, while the signals for Gaa1p and Cdc91p remained extremely low and undetectable, respectively $[20,30]$. Yet, as judged by silver staining, similar amounts of Gpi8p, Gpi16p and Gaa1p were present in the detergent purified yeast transamidase complex [7]. Also, genomic tagging of $C D C 91$ with GFP resulted in ER-localized fluorescence that was readily detected by light microscopy [18]. Thus, it is likely that, as in mammalian cells, yeast Gaa1p, Gpi8p, and Gpi16p are present at comparable amounts and that yeast cells also contain substantial amounts of Cdc91p.

As had been shown for Gpi8p through blue native gel electrophoresis [7], the fractionation of the endogenous detergent-solubilized transamidase complex over a glycerol density gradient (Figure 3) indicates that there were no free Gpi8p and Gpi16p subunits present, implying that the bulk of these proteins is contained within the previously purified Gpi8p-Gpi16p-Gaa1p complex. Intriguingly however, a large part of Gpi17p remained in the low density fractions of the gradient. This finding is fully consistent with the recent data of Ghaemmaghami et al. [20] suggesting that Gpi17p may be in excess of the other transamidase subunits. In agreement with this, Grimme et al. [18] found that Cdc91p-GST expressed from a single copy vector under its own promoter brings down only a small fraction (an estimated 10\%) of Gpi17p, whereas strong overexpression of Cdc91p-GST allowed to co-precipitate Gpi17p quantitatively. This suggests a direct interaction between Cdc91p and Gpi17p. Indeed, based on these findings and on genetic interactions these authors suggest the possibility of the existence of two subcomplexes, one containing Cdc91p and Gpi17p, the other one containing Gpi8p, Gpi16p and Gaa1p. Our data give additional support to this concept, since Gpi17p is not found in the affinity purified Gpi8p-Gaa1p-Gpi16p complex (Fig. 2) and only a small fraction of Gpi8p and Gpi16p is associated with Gpi17p in co-precipitation experiments (Fig. 3B-D). These data argue that the interaction of Gpi17p with the Gaa1p-Gpi8p-Gpi16p core is either very weak or that at any given time only a small fraction of this core is in contact with Gpi17p. Neither Gpi17p nor Cdc91p contain KKXX/KXKXX motifs for ER retrieval, but Gpi17p-GFP has nevertheless been localized to the ER [30], indicating that it is properly localized to interact with Gpi8p and Gpi16p, which latter carry such motifs. The failure to obtain dominant negative alleles of Gpi17p in a saturating screen is also compatible with the 
idea that, in yeast, Gpi17p may be able to exert its GPI anchoring function by interacting only weakly or transiently with the other transamidase subunits. Yet, this negative result by no means can prove this point. Indeed, our failure to get dominant negative alleles may simply be due to the difficulty in getting non-functional alleles (Table 2). It is well possible that these mutations nevertheless compromise the GPI anchoring activity in a subtle way that is not detected in ordinary growth assays. It also is possible that Gpi17p fulfills two different functions, and that the regions that were mutated in our experiments are required for some unknown, nonessential function whereas other regions may be required for the essential role of Gpi17p in GPI anchoring.

Notwithstanding all the arguments against a firm interaction between Gpi17p and the GaalpGpi8p-Gpi16p complex, it is clear that yeast Gpi17p, similar to its human PIG-S homologue, plays an important role in GPI anchoring, since its partial depletion leads to the accumulation of $\mathrm{CP} 2$, the mature GPI lipid of yeast $([8,18]$, data not shown), a phenomenon that is highly characteristic for transamidase mutants in yeast. PIG-T is aiding the formation of the acylenzyme intermediate [8], and to accomplish this in yeast, the Cdc91p-Gpi17p subcomplex may, at a certain stage, have to get into contact with the Gpi8p-Gpi16p-Gaa1p subcomplex. Thus, at a given time, only the few Cdc91p-Gpi17p subcomplexes, which are in contact with the Gpi8p-Gpi16p-Gaa1p subcomplex, may be active in GPI anchoring. The studies on the stability of transamidase subunits upon depletion of single subunits seem to indicate a certain degree of mutual stabilization, as Gpi17p seems to stabilize Gpi8p and to be stabilized by Gaa1p, and hence may also be indicative of some interactions. However, it seems that the stability can not easily be interpreted, since the stability of subunits may be dependent on the intensity of the ongoing UPR, and possibly on the rate at which cells loose viability upon transamidase depletion.

The molecular weights of all five known yeast transamidase subunits add up to about $330 \mathrm{kDa}$ but, since Gpi17p does not seem to be part of the complex, we presently only can account for $270 \mathrm{kDa}$. Gpi8p was present in a 430 - $650 \mathrm{kDa}$ band in blue native gel electrophoresis [7]. In this context we tested whether the complex contains multiple copies of some subunits. Using a diploid strain in which one of the two chromosomal GPI8 genes was modified by the Cterminal insertion of a TAP tag [20], we found that the untagged wt Gpi8p did not copurify with the digitonin-solubilized and affinity purified TAP-tagged Gpi8p, suggesting that the transamidase complex contains one single subunit of Gpi8p (not shown). By the same procedure, using the corresponding TAP-tagged strain, we found that the yeast complex contains a single subunit of Gpi16p. Using a similar approach, it has been shown that the 
human transamidase complex contains a single subunit of GPI8, PIG-T (hGpi16) and GAA1 [16]. Moreover, it was shown that there is no association of the yeast transamidase complex with the translocation pore or the oligosaccharyltransferase complex [7]. Thus, for the moment we are inclined to believe that the use of soluble proteins as standards in native blue electrophoresis may not be appropriate for the weight determination of membrane complexes containing large amounts of bound detergent micelles and that the apparent molecular weight of 430-650 $\mathrm{kDa}$ for the yeast transamidase complex may be an overestimation.

Using a GPI17-TAP/GPII7 diploid strain we also found that tagged Gpi17p-TAP was not associated with untagged Gpi17p (not shown). Thus, although Gpi17p seems to be associated with some high molecular weight complex in blue native gel electrophoresis, this complex is not stable during glycerol gradient fractionation (Fig. 3).

While further experiments are required to identify potential interaction partners of Gpi17p, our present findings point to the existence of two GPI-transamidase subcomplexes, one containing the Gpi8p-Gpi16p-Gaa1p core and the other one containing Gpi17p and probably Cdc91p. This new concept raises the possibility that the GPI anchoring process can be subdivided into several steps some of which may be achieved by separate subcomplexes.

\section{Acknowledgements}

We thank Drs. Susan Michaelis, Markus Aebi, Thomas Sommer, Rosine Haguenauer-Tsapis, and Régine Bosson for the gift of strains. This work was supported by grant 31-67188.01 from the Swiss National Science Foundation. 


\section{Tables}

Table 1. Saccharomyces cerevisiae strains

\begin{tabular}{|c|c|c|}
\hline Strain & Genotype/Plasmid & Reference \\
\hline W303-1B & $\begin{array}{l}\text { W303-1B MAT } \alpha \text { ade2-1 can1-100 ura3-1 leu2-3,112 trp1-1 } \\
\text { his3- trp1-1 his3-11,15 }\end{array}$ & \\
\hline FBY735 & $\begin{array}{l}\text { MATa his } 3 \Delta 1 \text { leu } 2 \Delta 0 \text { lys } 2 \Delta 0 \text { ura3 } \Delta 0 \text { gpil6::kanMX4 } \\
\text { containing YCplac111- } P_{G A L I}-G P I 16\end{array}$ & [7] \\
\hline FBY164 & $\begin{array}{l}\text { MAT } \mathbf{\alpha} \text { ade2-1 leu2-3,112 his3-11,15 gpi8::kanMX2 ura3- } \\
1:: U R A 3-P_{G A L I 10^{-}} \text {GPI8 }\end{array}$ & [13] \\
\hline Y24270 & 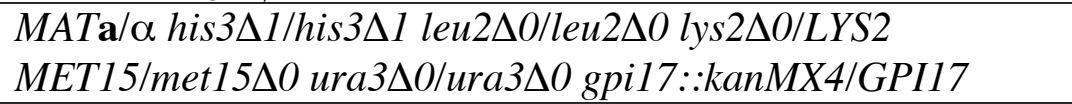 & EUROSCARF \\
\hline FBY7310 & 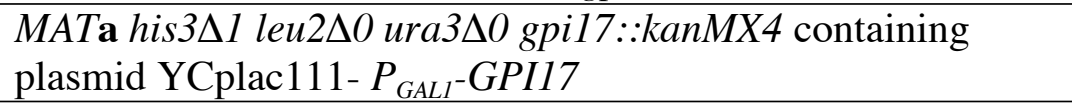 & This study \\
\hline FBY739 & $\begin{array}{l}\text { MATa his } 3 \Delta 1 \text { leu } 2 \Delta 0 \text { ura3 } 30 \text { gpil7::kanMX4 containing } \\
\text { plasmid pYES-GPII7 }\end{array}$ & This study \\
\hline FBY455 & $\begin{array}{l}\text { MAT } \alpha \text { ade } 2-1 \text { can1-100 ura3-1 leu2-3,112 trp1-1 his3- trp1-1 } \\
\text { his3-11,15 containing plasmid CUP195-GPII7 }\end{array}$ & This study \\
\hline FBY459 & 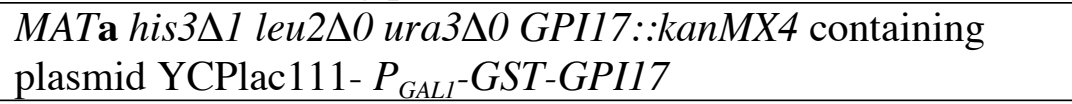 & This study \\
\hline FBY810a & 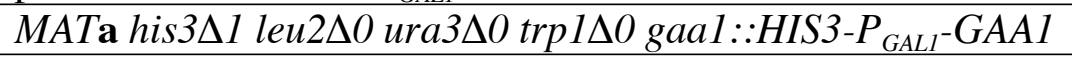 & This study \\
\hline $\begin{array}{l}\text { SM2561= } \\
\text { MHY500 }\end{array}$ & MATa his3 leu2 ura3 lys 2 trpl & {$[31]$} \\
\hline $\begin{array}{l}\text { SM 2564= } \\
\text { MHY552 }\end{array}$ & 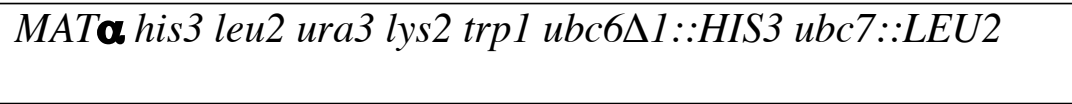 & [31] \\
\hline $\begin{array}{l}\text { SM } 2682= \\
\text { MHY623 }\end{array}$ & $\begin{array}{l}\text { MAT } \mathbf{\alpha} \text { his3 } 200 \text { leu2-3,112 ura3-52 lys2-801 trpl-1 } \\
\text { doa4::LEU2 }\end{array}$ & {$[31]$} \\
\hline $\begin{array}{l}\text { SM 2456= } \\
\text { MS10 }\end{array}$ & MATa ura3-52 leu2-3,112 ade2-101 & [31] \\
\hline $\begin{array}{l}\text { SM 2537= } \\
\text { MS1907 }\end{array}$ & MATa ade2-101 leu2-3,112 ura3-52 pep4::LEU2 & {$[31]$} \\
\hline SS0328 & MATa ade2-101 his $3 \Delta 200$ lys2-801 ura3-52 & M. Aebi, ETHZ \\
\hline YG0840 & 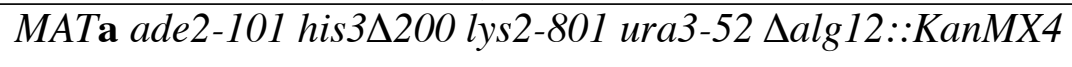 & M. Aebi, ETHZ \\
\hline YG0618 & 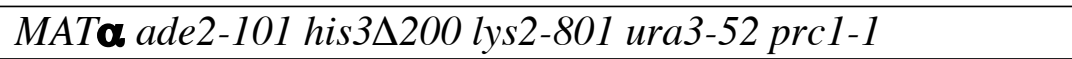 & M. Aebi, ETHZ \\
\hline YG0807 & 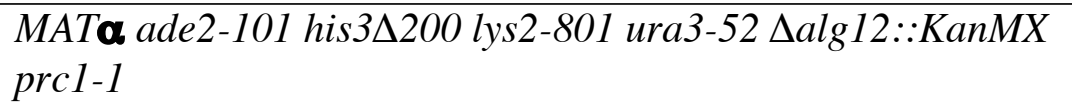 & M. Aebi, ETHZ \\
\hline YG0746 & 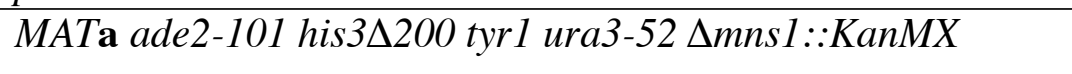 & M. Aebi, ETHZ \\
\hline YJU82 & 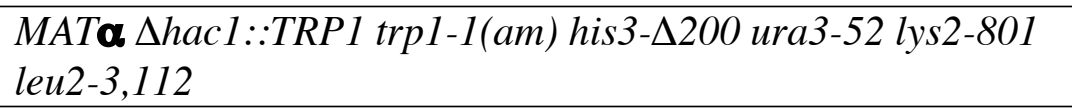 & [32] \\
\hline YW01 & 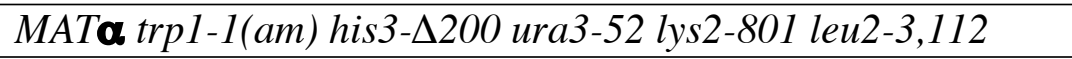 & {$[32]$} \\
\hline YJU95 & $\begin{array}{l}\text { MATa } \Delta \text { hrd1::TRP1 trp1-1(am) his3- } \Delta 200 \text { ura3-52 lys2-801 } \\
\text { leu2-3,112 }\end{array}$ & {$[32]$} \\
\hline
\end{tabular}




\begin{tabular}{|c|c|c|}
\hline YRF13 & 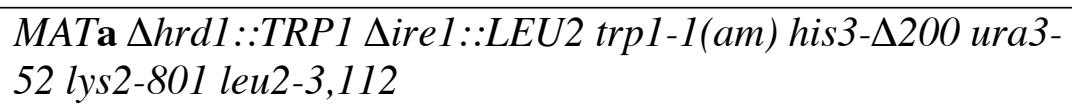 & [32] \\
\hline YJU85 & 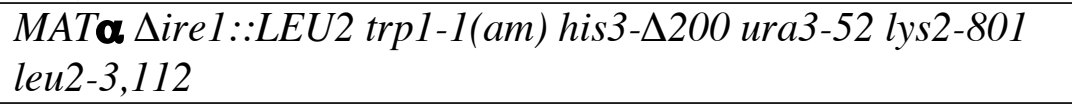 & {$[32]$} \\
\hline YTX144 & 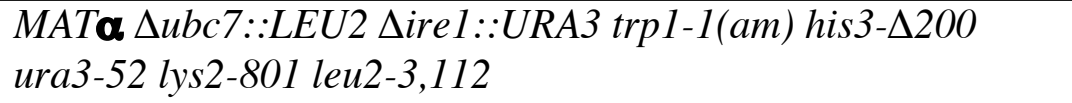 & {$[32]$} \\
\hline YTX244 & 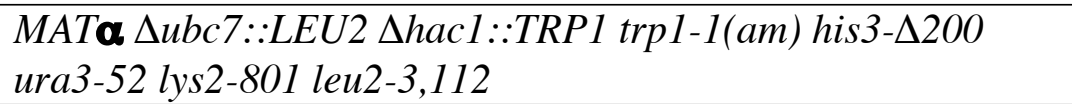 & [32] \\
\hline YTX237 & $\begin{array}{l}\text { MATa } \triangle u b c 1: \because H I S 3 \Delta u b c 7:: L E U 2 \text { trp1-1(am) his3- } \Delta 200 \\
\text { ura3-52 lys2-801 leu2-3,112 }\end{array}$ & [32] \\
\hline YPH 499 & 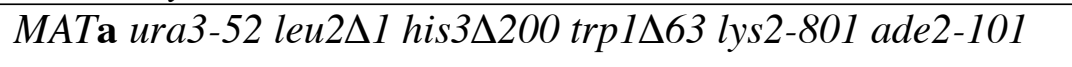 & [33] \\
\hline CMY 763 & 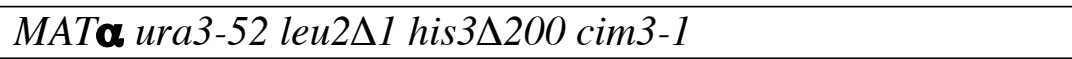 & [33] \\
\hline CMY 765 & 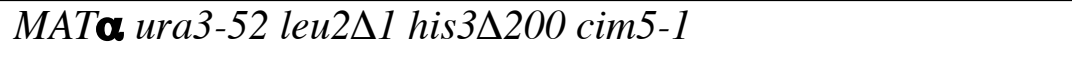 & [33] \\
\hline WCG 4a & MATa ura3 leu2-3,112 his3-115 & {$[33]$} \\
\hline $\begin{array}{l}\text { WCG 4- } \\
11 / 22 \mathrm{a}\end{array}$ & MATa ura3 leu2-3,112 his3-115 pre1-1 pre2-2 & [33] \\
\hline $27061 b$ & MATa ura3 trp1 & [33] \\
\hline $27064 b$ & MATa ura3 trpl npil & {$[33]$} \\
\hline FBY656 & $\begin{array}{l}\text { MATa ade2-1 ura3-1 leu2-3,112 trp1-1 his3-11,15 lys } \\
\text { gpi8::kanMX2 containing YCplac22-GST-GPI8 }\end{array}$ & [7] \\
\hline $\begin{array}{l}\text { GPI17- } \\
\text { TAP }^{1}\end{array}$ & 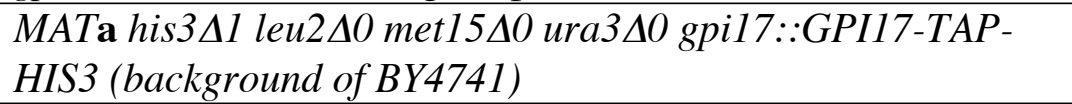 & {$[20]$} \\
\hline BY4741 & 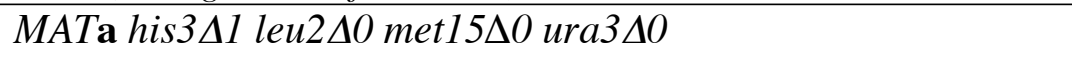 & EUROSCARF \\
\hline
\end{tabular}

$\mathrm{P}=$ promoter. ${ }^{1}$ TAP strains contain an in frame insert of a TAP tag at the C-terminus of a chromosomal gene. 
Table 2. Functionality of different GPII7 alleles

\begin{tabular}{|c|c|c|c|}
\hline $\begin{array}{l}\text { Mutation in } \\
\text { conserved } \\
\text { residues }\end{array}$ & $\begin{array}{l}\text { Degree of conservation (other amino } \\
\text { acids in that position) }\end{array}$ & $\begin{array}{l}\text { Dominant } \\
\text { negative after } \\
\text { retransfection }\end{array}$ & Funct-ional \\
\hline G22S & 10/11 (A) & no & yes \\
\hline W26R & 9/11 (L) & no & yes \\
\hline $\begin{array}{l}\mathrm{Y} 27 \mathrm{H} \\
\mathrm{I} 42 \mathrm{~T}\end{array}$ & $\begin{array}{l}\text { 1/11 (WLH) } \\
7 / 11(\mathrm{MS})\end{array}$ & no & yes \\
\hline $\begin{array}{l}\mathrm{A} 35 \mathrm{~T} \\
\mathrm{C} 13 \mathrm{Y}\end{array}$ & $\begin{array}{l}5 / 11(\mathrm{VS}) \\
1 / 11(\mathrm{FASYL})\end{array}$ & no & yes \\
\hline P38L & $11 / 11$ & no & yes \\
\hline $\begin{array}{l}\text { L212F } \\
\text { Y215F } \\
\text { F219S } \\
\text { T230I }\end{array}$ & $\begin{array}{l}\text { 1/11 (VAIDGT) } \\
\text { 7/11 (FLMA) } \\
\text { 5/11 (WVIL) } \\
\text { 6/11 (SNE, 8/11have N-glycosylation site } \\
\text { N228 conserved) }\end{array}$ & no & no \\
\hline F222S & 1/11 (ARKELQD) & no & yes \\
\hline $\mathbf{K}^{28 A^{\S}}$ & 9/11 (RF) & no & yes \\
\hline $\mathbf{R 3 4 A}^{\S}$ & 11/11 & no & yes \\
\hline \multicolumn{4}{|c|}{$\begin{array}{l}\text { The } 11 \text { full length GPII7 homologues compared at } \\
\text { http://mendel.imp.univie.ac.at/SEQUENCES/gpi-biosynthesis/ are EAA07616.1 Anopheles }\end{array}$} \\
\hline \multicolumn{4}{|c|}{ gambiae, NP_187374.1 Arabidopsis thaliana, AN5150.1 Aspergillus nidulans, T24913 } \\
\hline \multicolumn{4}{|c|}{$\begin{array}{l}\text { Caenorhabditis elegans, orf19.6379.prot Candida albicans, AAL27645.1 Drosophila } \\
\text { melanogaster, NP_149975.1 Homo sapiens, XP_203412.1 Mus musculus, XP_325888.1 }\end{array}$} \\
\hline \multicolumn{4}{|c|}{ Neurospora crassa, BAB92563.1 Oryza sativa, NP_010722.1 Saccharomyces cerevisiae. } \\
\hline
\end{tabular}




\section{Figure legends}

FIG. 1. Gpi17p is a 63kDa integral membrane glycoprotein. Antisera were raised against hydrophilic fragments or peptides of Gpi17p in rabbits. A, affinity purified anti-peptide antibodies (lanes 1-3) or rabbit anti-protein antibodies (lanes 4-9) were used to detect antigens by Western blotting in cell lysates from wt cells (BY4741, lane 1), or gpi17A cells containing plasmids allowing the overexpression of Gpi17p, namely YCplac111-GPII7 (lanes 2, 3),

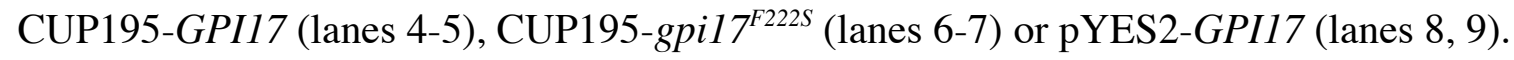
Strains were grown in BCS versus copper or glucose versus galactose, to repress or induce Gpi17p, respectively. B, extracts from wt cells (W303-1B, lanes 1, 2) or cells overexpressing Gpi17p (FBY455, lanes 3, 4) were treated with Endoglycosidase H or mock incubated and blotted with anti-Gpi17p antibody. C, GPI17-TAP cells were treated with zymolyase, spheroplasts were lysed and aliquots of the lysate were incubated for $30 \mathrm{~min}$ at $4^{\circ} \mathrm{C}$ with $1 \mathrm{M}$ $\mathrm{NaCl}, 0.11 \mathrm{M} \mathrm{Na}_{2} \mathrm{CO}_{3}, 1 \%$ Triton X-100 or $1 \%$ SDS. Subsequently, membranes were sedimented by ultracentrifugation. Proteins of supernatants $(\mathrm{S})$ and pellets $(\mathrm{P})$ were precipitated with trichloroacetic acid, processed for SDS-PAGE/Western blotting, and probed with anti-protein A antibodies.

FIG. 2. Purified yeast transamidase complex does not contain Gpi17p. A, a digitonin extract from gpi8$\Delta$ cells harboring a GST-tagged Gpi8p (FBY656) was used to purify the transamidase complex on a glutathione-Sepharose column and samples of the starting lysate, the run-through, the washes and the material eluted with glutathione were analyzed by Western blotting using affinity-purified anti-Gpi17p antibodies. B, digitonin solubilized membrane proteins from FBY656 were run in blue native electrophoresis (7) and probed with the indicated antibodies. Lane 1: Anti-Gpi17p; lane 2: secondary antibody only; lanes 3-4: anti-Gpi8p; Lanes 3 - 4 were stripped, cut in half and reprobed with either anti-Gpi17p (lane 3') or anti-Gpi8p (lane 4'). In lanes 1, 2 and 3' - 4', the secondary antibody was goat antirabbit IgG-alkaline phosphatase, in lanes 3 - 4 it was goat anti-rabbit IgG-peroxidase.

FIG. 3. Gpi17p interacts only transiently with digitonin solubilized GPI-transamidase complexes. A, 1\% digitonin-solubilized membrane proteins from wt cells (W303-1B) were treated with DNAse and centrifuged (100’000 g, $45 \mathrm{~min})$ to remove the insoluble material. The lysate (L) was layered on top of a $12 \%$ to $32 \%$ glycerol density gradient (in $0.2 \%$ 
digitonin), which was centrifuged at $175^{\prime} 000 \mathrm{~g}$ for $15 \mathrm{~h}$. Twelve equal fractions as well as a small pellet $(\mathrm{P})$ were recovered and processed for Western blotting. $\mathbf{B}$, membranes of cells overexpressing GST-tagged Gpi17p (FBY459) were isolated and solubilized with 1\% digitonin. The transamidase complex was affinity-precipitated using glutathione-Sepharose $4 \mathrm{~B}$ and the bound material was eluted with $100 \mathrm{mM}$ reduced glutathione (E); the starting lysate (Lysate) as well as the eluate (E) were processed for Western blotting. The corresponding $\mathrm{OD}_{600}$ units of starting cells indicate the amount of material loaded. $\mathbf{C}$, membranes of GPI17-TAP or wt cells were lysed in digitonin (C) or NP-40 (D), the transamidase complex was pulled down using IgG-Sepharose and resolved on SDS-PAGE. In C, Gpi17p-TAP was detected using anti-Protein A antibodies, while Gpi8p and Gpi16p were detected with anti-Gpi8p and anti-Gpi16p antibodies. In D, the membrane was probed only with anti-Gpi16p, which detects both Gpi16p and Gpi17-TAP because the TAP tag binds the Fc domain of antibodies.

FIG. 4. GPI-transamidase subunits mutually stabilize each other. Strains FBY164, FBY810a, FBY735 and FBY7310 containing their single copy of GPI8, GAA1, GPI16 or GPI17, respectively, under the control of the GAL1 promoter were shifted from galactose to glucose and further grown for $0,5,15$ and 40 hours for depletion of products of the genes indicated on top of each column (depleted proteins boxed). Proteins were extracted, separated on SDS-PAGE and probed by Western blot with antibodies against the proteins indicated to the right. All lanes contained equal amounts of porin, which was used as a loading control (not shown).

FIG. 5. Stability of GPI-transamidase subunits in ERAD vacuolar degradation mutants. The indicated strains were grown exponentially in YPDUA at $30^{\circ} \mathrm{C}$, proteins were extracted, separated on SDS-PAGE, blotted and probed with antibodies against the proteins indicated to the right. $\operatorname{Cim} 3-1$ and $\operatorname{cim} 5-1$ cells were grown at $24^{\circ}$ and shifted to nonpermissive temperature $\left(37^{\circ} \mathrm{C}\right)$ during 1 or $4 \mathrm{~h}$ before extraction. 


\section{References}

[1]I.W. Caras, G.N. Weddell, M.A. Davitz, V. Nussenzweig, D.W.J. Martin, Signal for attachment of a phospholipid membrane anchor in decay accelerating factor, Science 238 (1987) 1280-1283.

[2]P. Moran, I.W. Caras, Fusion of sequence elements from non-anchored proteins to generate a fully functional signal for glycophosphatidylinositol membrane anchor attachment, $\mathrm{J}$ Cell Biol 115 (1991) 1595-1600.

[3]L.D. Gerber, K. Kodukula, S. Udenfriend, Phosphatidylinositol glycan (PI-G) anchored membrane proteins. Amino acid requirements adjacent to the site of cleavage and PI-G attachment in the COOH-terminal signal peptide, J Biol Chem 267 (1992) 1216812173.

[4]C. Nuoffer, A. Horvath, H. Riezman, Analysis of the sequence requirements for glycosylphosphatidylinositol anchoring of Saccharomyces cerevisiae Gas1 protein, J Biol Chem 268 (1993) 10558-10563.

[5]D. Hamburger, M. Egerton, H. Riezman, Yeast Gaa1p is required for attachment of a completed GPI anchor onto proteins, J Cell Biol 129 (1995) 629-639.

[6]M. Benghezal, A. Benachour, S. Rusconi, M. Aebi, A. Conzelmann, Yeast Gpi8p is essential for GPI anchor attachment onto proteins, EMBO J 15 (1996) 6575-6583.

[7]P. Fraering, I. Imhof, U. Meyer, J.M. Strub, D.A. van, C. Vionnet, A. Conzelmann, The GPI transamidase complex of Saccharomyces cerevisiae contains Gaa1p, Gpi8p, and Gpi16p, Mol Biol Cell 12 (2001) 3295-3306.

[8]K. Ohishi, N. Inoue, T. Kinoshita, PIG-S and PIG-T, essential for GPI anchor attachment to proteins, form a complex with GAA1 and GPI8, EMBO J 20 (2001) 4088-4098.

[9]Y. Hong, K. Ohishi, J.Y. Kang, S. Tanaka, N. Inoue, J. Nishimura, Y. Maeda, T. Kinoshita, Human PIG-U and yeast Cdc91p are the fifth subunit of GPI transamidase that attaches GPI-anchors to proteins, Mol Biol Cell 14 (2003) 1780-1789.

[10]S. Vainauskas, Y. Maeda, H. Kurniawan, T. Kinoshita, A.K. Menon, Structural requirements for the recruitment of Gaa1 into a functional glycosylphosphatidylinositol transamidase complex, J Biol Chem 277 (2002) 30535-30542.

[11]S. Vainauskas, A.K. Menon, A conserved proline in the last transmembrane segment of Gaa1 is required for glycosylphosphatidylinositol (GPI) recognition by GPI transamidase, J Biol Chem 279 (2004) 6540-6545. 
[12]J.M. Chen, N.D. Rawlings, R.A. Stevens, A.J. Barrett, Identification of the active site of legumain links it to caspases, clostripain and gingipains in a new clan of cysteine endopeptidases, FEBS Lett 441 (1998) 361-365.

[13]U. Meyer, M. Benghezal, I. Imhof, A. Conzelmann, Active site determination of Gpi8p, a caspase-related enzyme required for glycosylphosphatidylinositol anchor addition to proteins, Biochemistry 39 (2000) 3461-3471.

[14]K. Ohishi, N. Inoue, Y. Maeda, J. Takeda, H. Riezman, T. Kinoshita, Gaa1p and gpi8p are components of a glycosylphosphatidylinositol (GPI) transamidase that mediates attachment of GPI to proteins, Mol Biol Cell 11 (2000) 1523-1533.

[15]X. Kang, A. Szallies, M. Rawer, H. Echner, M. Duszenko, GPI anchor transamidase of Trypanosoma brucei: in vitro assay of the recombinant protein and VSG anchor exchange, J Cell Sci 115 (2002) 2529-2539.

[16]K. Ohishi, K. Nagamune, Y. Maeda, T. Kinoshita, Two subunits of glycosylphosphatidylinositol transamidase, GPI8 and PIG-T, form a functionally important intermolecular disulfide bridge, J Biol Chem 278 (2003) 13959-13967.

[17]B. Eisenhaber, S. Maurer-Stroh, M. Novatchkova, G. Schneider, F. Eisenhaber, Enzymes and auxiliary factors for GPI lipid anchor biosynthesis and post-translational transfer to proteins, Bioessays 25 (2003) 367-385.

[18]S.J. Grimme, X.D. Gao, P.S. Martin, K. Tu, S.E. Tcheperegine, K. Corrado, A.E. Farewell, P. Orlean, E. Bi, Deficiencies in the endoplasmic reticulum (ER)-membrane protein Gablp perturb transfer of glycosylphosphatidylinositol to proteins and cause perinuclear ER-associated actin bar formation, Mol Biol Cell 15 (2004) 2758-2770.

[19]K. Nagamune, K. Ohishi, H. Ashida, Y. Hong, J. Hino, K. Kangawa, N. Inoue, Y. Maeda, T. Kinoshita, GPI transamidase of Trypanosoma brucei has two previously uncharacterized (trypanosomatid transamidase 1 and 2) and three common subunits, Proc Natl Acad Sci U S A 100 (2003) 10682-10687.

[20]S. Ghaemmaghami, W.K. Huh, K. Bower, R.W. Howson, A. Belle, N. Dephoure, E.K. O'Shea, J.S. Weissman, Global analysis of protein expression in yeast, Nature 425 (2003) 737-741.

[21]F. Sherman, Getting started with yeast, Methods Enzymol 350 (2002) 3-41.

[22]M.S. Longtine, A.r. McKenzie, D.J. Demarini, N.G. Shah, A. Wach, A. Brachat, P. Philippsen, J.R. Pringle, Additional modules for versatile and economical PCR-based gene deletion and modification in Saccharomyces cerevisiae, Yeast 14 (1998) 953-961. 
[23]T. Hottiger, P. Furst, G. Pohlig, J. Heim, Physiological characterization of the yeast metallothionein (CUP1) promoter, and consequences of overexpressing its transcriptional activator, ACE1, Yeast 10 (1994) 283-296.

[24]V.V. Kushnirov, Rapid and reliable protein extraction from yeast, Yeast 16 (2000) 857 860.

[25]I. Imhof, I. Flury, C. Vionnet, C. Roubaty, D. Egger, A. Conzelmann, Glycosylphosphatidylinositol (GPI) proteins of Saccharomyces cerevisiae contain ethanolamine phosphate groups on the alpha1,4-linked mannose of the GPI anchor, J Biol Chem 279 (2004) 19614-19627.

[26]R. Menzel, E. Kargel, F. Vogel, C. Bottcher, W.H. Schunck, Topogenesis of a microsomal cytochrome $\mathrm{P} 450$ and induction of endoplasmic reticulum membrane proliferation in Saccharomyces cerevisiae, Arch Biochem Biophys 330 (1996) 97-109.

[27]R. Menzel, F. Vogel, E. Kargel, W.H. Schunck, Inducible membranes in yeast: relation to the unfolded-protein-response pathway, Yeast 13 (1997) 1211-1229.

[28]K.J. Travers, C.K. Patil, L. Wodicka, D.J. Lockhart, J.S. Weissman, P. Walter, Functional and genomic analyses reveal an essential coordination between the unfolded protein response and ER-associated degradation, Cell 101 (2000) 249-258.

[29]R.Y. Hampton, ER-associated degradation in protein quality control and cellular regulation, Curr Opin Cell Biol 14 (2002) 476-482.

[30]W.K. Huh, J.V. Falvo, L.C. Gerke, A.S. Carroll, R.W. Howson, J.S. Weissman, E.K. O'Shea, Global analysis of protein localization in budding yeast, Nature 425 (2003) 686-691.

[31]D. Loayza, A. Tam, W.K. Schmidt, S. Michaelis, Ste6p mutants defective in exit from the endoplasmic reticulum (ER) reveal aspects of an ER quality control pathway in Saccharomyces cerevisiae, Mol Biol Cell 9 (1998) 2767-2784.

[32]R. Friedlander, E. Jarosch, J. Urban, C. Volkwein, T. Sommer, A regulatory link between ER-associated protein degradation and the unfolded-protein response, Nat Cell Biol 2 (2000) 379-384.

[33]J.M. Galan, V. Moreau, B. Andre, C. Volland, R. Haguenauer-Tsapis, Ubiquitination mediated by the Npi1p/Rsp5p ubiquitin-protein ligase is required for endocytosis of the yeast uracil permease, J Biol Chem 271 (1996) 10946-10952. 
A

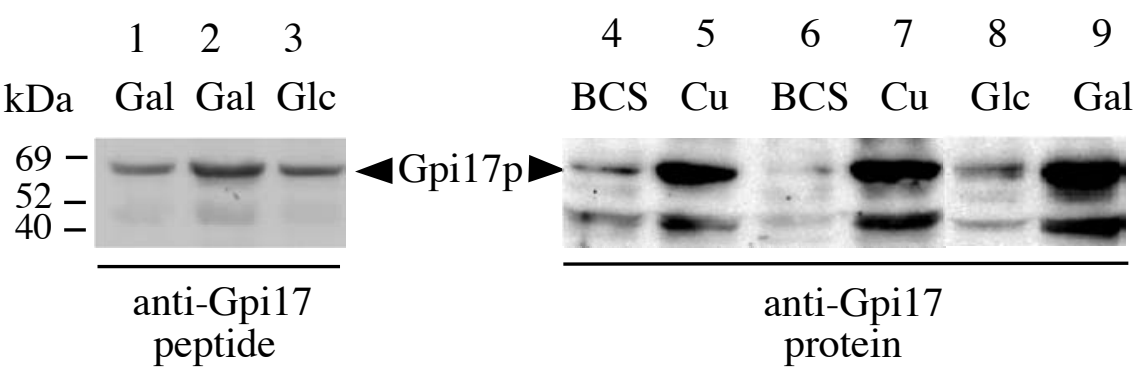

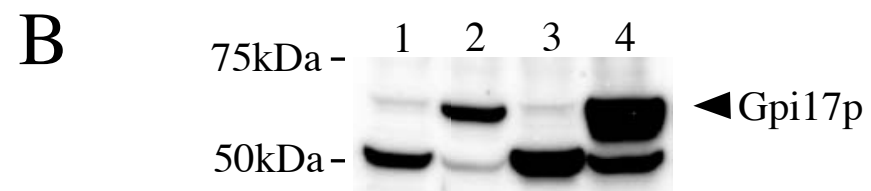

Endo $\mathrm{H}+-+-$

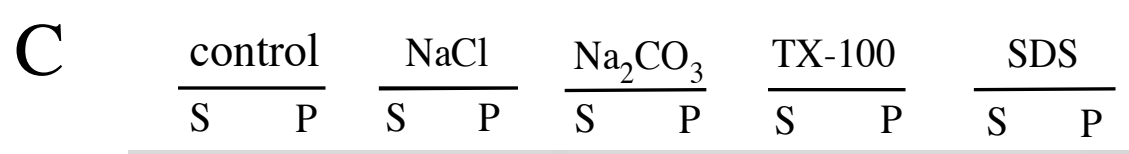

TAP-Gpi17p

(94 kDa)

Zhu et al., Fig. 1 
A

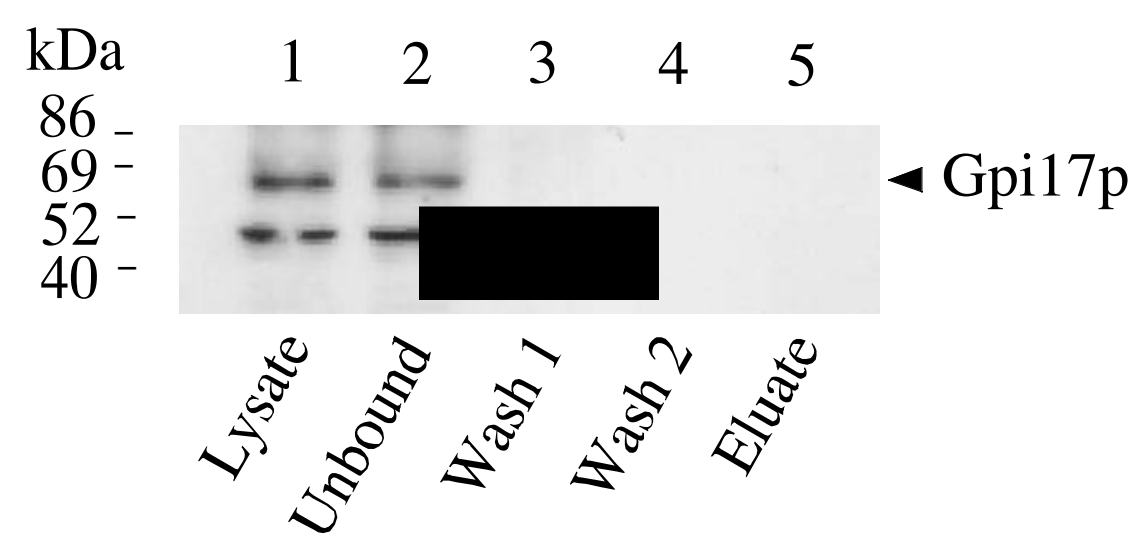

B

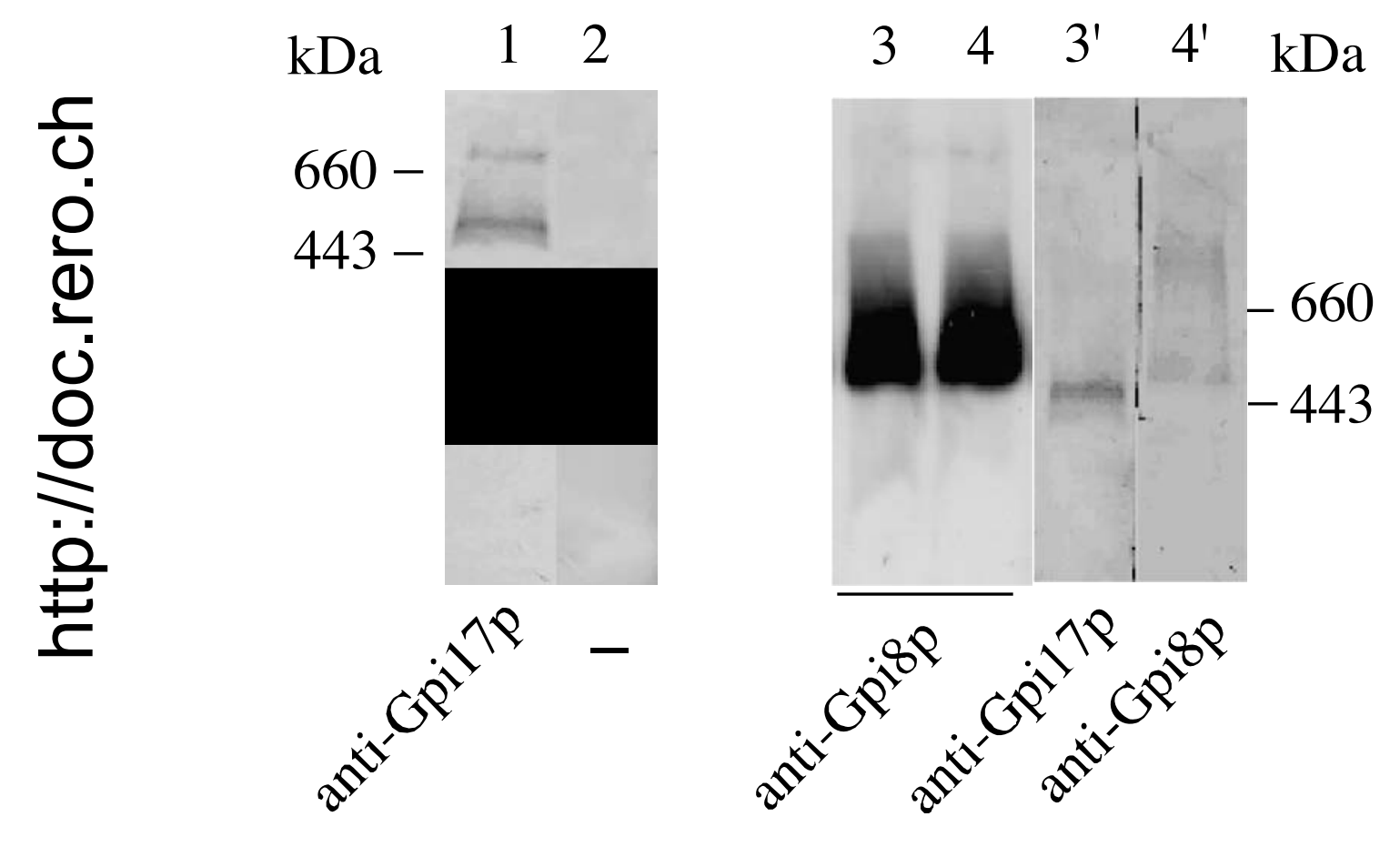

Zhu et al., Fig. 2 
A $12 \%$

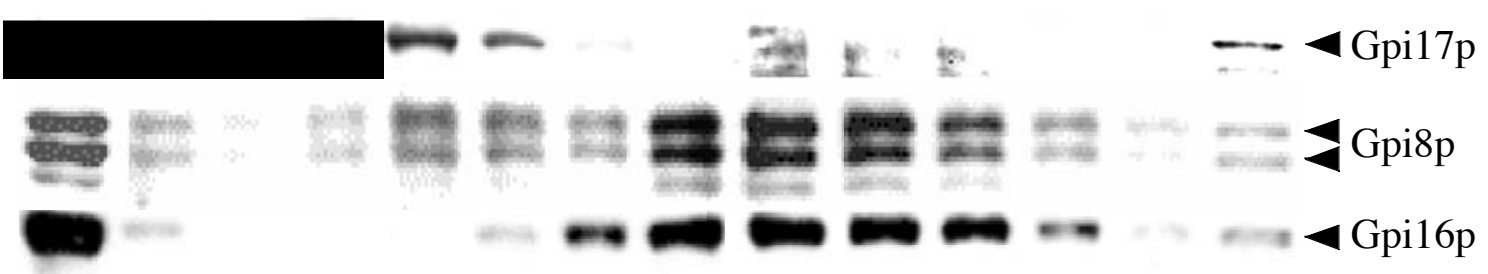

$\begin{array}{lllllllllllllll}\mathbf{L} & 1 & 2 & 3 & 4 & 5 & 6 & 7 & 8 & 9 & 10 & 11 & 12 & \mathbf{P} & \text { Fraction }\end{array}$

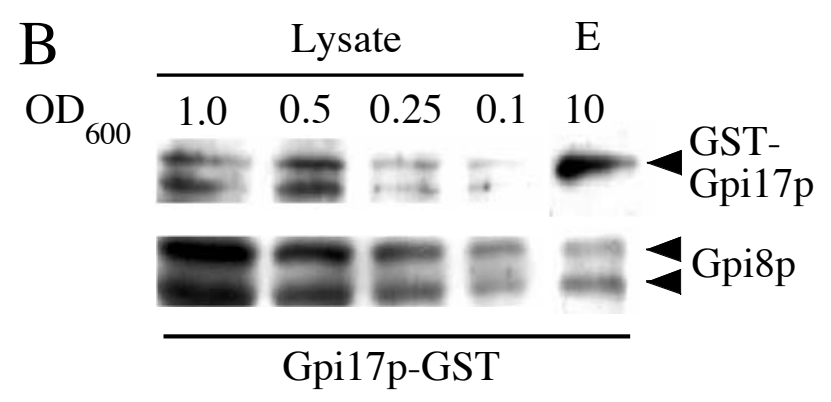

$\mathrm{D} \quad \mathrm{OD}_{600} \begin{array}{ccc}\mathrm{L} & \mathrm{E} \\ & 2.5\end{array}$

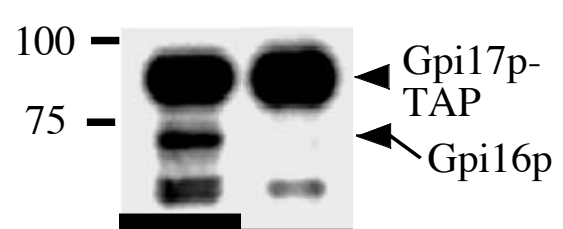

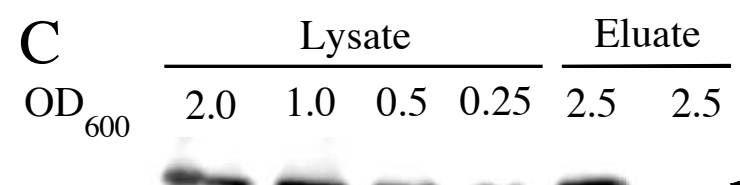

$50-$

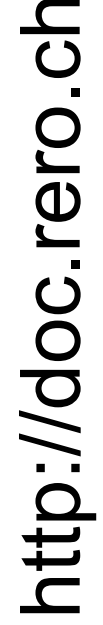

Zhu et al., Fig. 3 


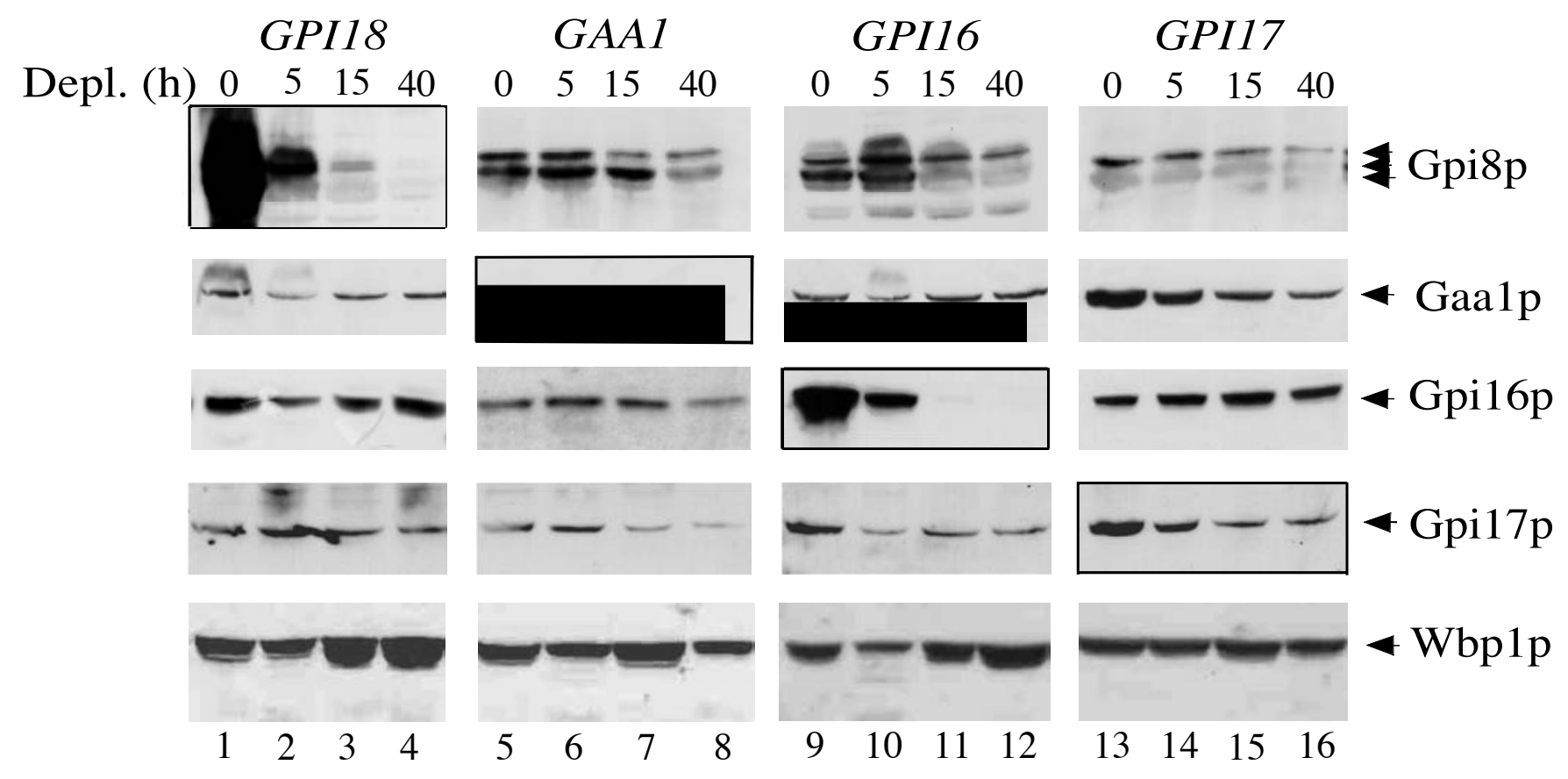

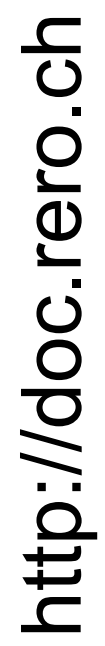

Zhu et al., Fig. 4 


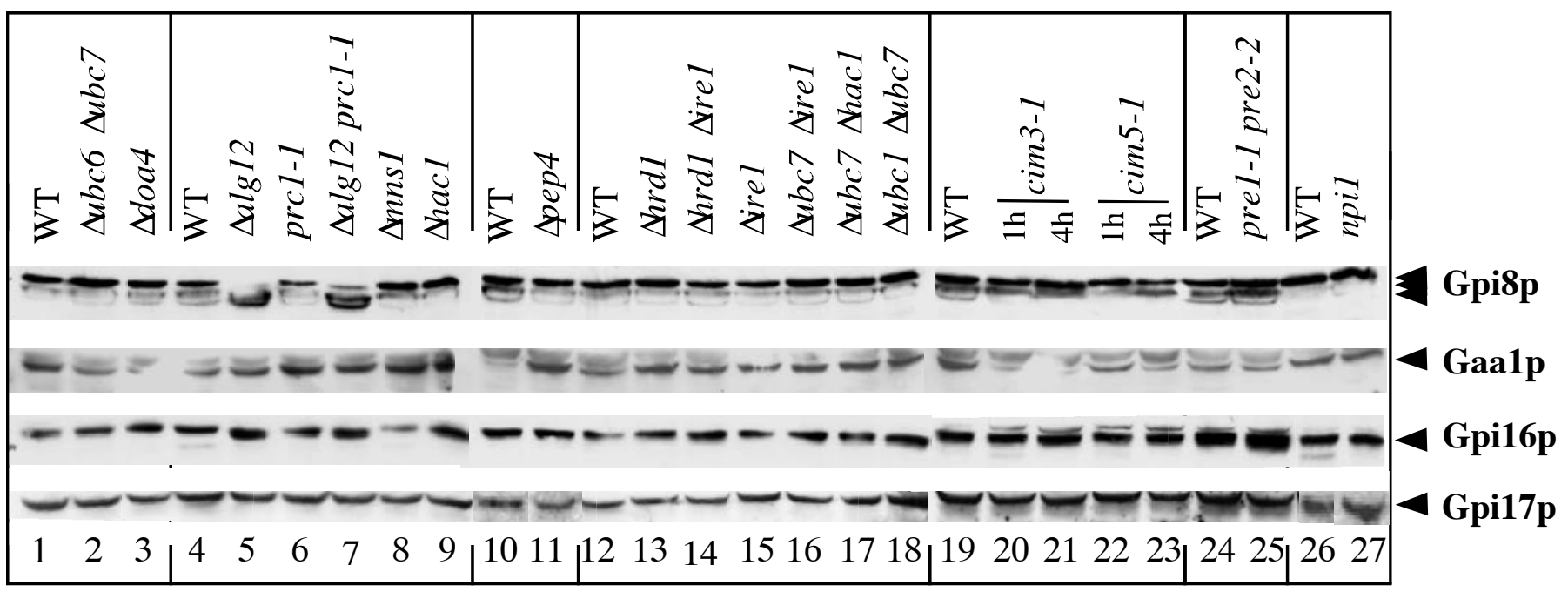

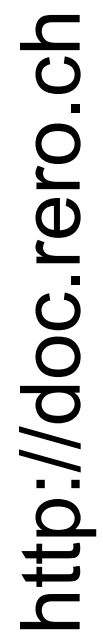

Zhu et al., Fig. 5 